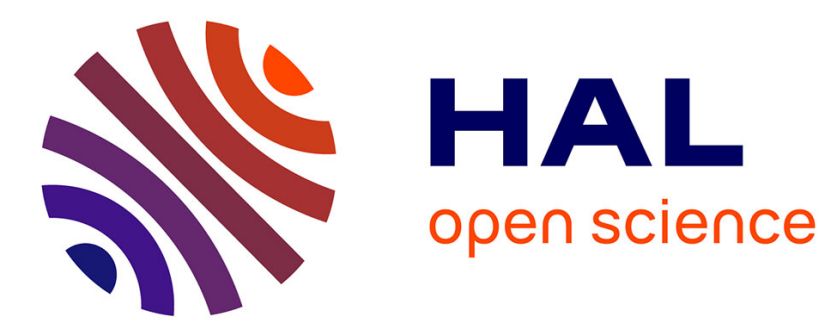

\title{
Model-Space Regularization and Fully Interpretable Algorithms for Postural Control Quantification
}

\author{
Alice Nicolaï, Julien Audiffren
}

\section{To cite this version:}

Alice Nicolaï, Julien Audiffren. Model-Space Regularization and Fully Interpretable Algorithms for Postural Control Quantification. COMPSAC 2018, Jul 2018, Tokyo, Japan. hal-01944541

\author{
HAL Id: hal-01944541 \\ https://hal.science/hal-01944541
}

Submitted on 4 Dec 2018

HAL is a multi-disciplinary open access archive for the deposit and dissemination of scientific research documents, whether they are published or not. The documents may come from teaching and research institutions in France or abroad, or from public or private research centers.
L'archive ouverte pluridisciplinaire HAL, est destinée au dépôt et à la diffusion de documents scientifiques de niveau recherche, publiés ou non, émanant des établissements d'enseignement et de recherche français ou étrangers, des laboratoires publics ou privés. 


\section{Model-Space Regularization and Fully Interpretable Algorithms for Postural Control Quantification}

\author{
Alice Nicolaï \\ Centre de Mathematiques et Leurs Applications \\ ENS Paris Saclay \\ Cachan, France \\ Email: alice.nicolai@cmla.ens-cachan.fr
}

\author{
Julien Audiffren \\ Exascale Infolab \\ Fribourg University \\ Fribourg, Switzerland
}

\begin{abstract}
As falls prevalence increases with the aging of the population, early detection of balance degradation is of great importance for efficient prevention and treatment. This work addresses the problem of quantifiying static balance with fully interpretable learning algorithms. Our approach relies on a heuristic based variant of the aggregation of weak classifiers constrained with a new model-space regularization combined with a family of interpretable features. In our experiments, these models outperforms their regular alternative, opening promising new research directions.
\end{abstract}

\section{Introduction}

In the recent years, Machine Learning methods and algorithms have been successfully used in a wide range of problems, ranging from robotics [1] to recommander systems [2]. In particular, possible applications in Healthcare and Medical Research have gained significant interest recently, as the ageing of the populations will increase the need for efficient and widely available healthcare and treatments [3]. Notably, the screening and early diagnosis of diseases and conditions is one of the focal point of attention for Machine Learning methods, and Deep Learning approaches have already lead to breakthtough results in Medical Imagery (see e.g. [4]). Other methods have been proven valuable for computer-aided diagnosis [5], in particular for conditions where the early symptoms may be difficult to assess.

One domain in which early diagnosis is key is balance deterioration. Indeed, such impairments strongly increase the risk of falling, which can have devastating consequences - including serious injuries, dependency and death - particularly for the elderly population [6]. Consequently, fall prevention in the elderly population is of paramount importance, particularly as each year more than a third of population 65 years-old and older faces a fall [7]. A key ingredient to fall prevention is the early detection of balance deterioration, which can then be treated by prevention strategies such as reeducation [8]. Accurate quantification of balance control can be of significant assistance to such detection, and can also be used to evaluate the performances of prevention and recovery strategies.
Quantifying Balance. Balance is achieved by a complex synergy of many biological systems, including the visual, proprioceptive, vestibular and central nervous system [9] - and therefore is difficult to thoroughly evaluate. Generally, balance is considered as the combination of a static and a dynamic component [10]. Static balance is defined as the capacity to stand still without falling whereas dynamic equilibrium is the ability to balance while in motion or when switching between positions, and both components can be tested to evaluate the risk of falling, through observational tests or measurements (see e.g. [11]).

Static Balance (also called Postural control) is generally considered as the easiest component to measure, as it does not require complex protocols and expensive sensors, and has been shown to be correlated to the risk of fall [11]. Postural control is generally measured by recording the displacement of the center of pressure $(\mathrm{CoP})$ - an approximation of the projection of the Center of Mass, see [12] - over time using a force platform [13]. The resulting signal is usually called a statokinesigram (in this work, a two dimensional signal), that has been shown to contain significant information on postural control [14] for a wide range of acquisition protocols, such as the Romberg's test [15], [16].

Related Works. The problem of quantifying postural control is hardly new, and numerous previous works have analyzed statokinesigrams with the aim of distinguishing between specific populations. The most commonly used approaches proceed as follows : a small group of scalar features are derived from the CoP trajectory - commonly used features include average speed, lateral variance and surface spanned by the trajectory (see e.g. [14]). Then a statistical analysis - generally a parametric statistical test - is applied to determine whether those features follow different distributions depending on the populations studied. For instance, in the seminal work of [14], it is shown that some features such as sway density peaks follow different distributions in parkinsonian, osteoporotic and a heatlhy populations; in [11] others descriptors such as length of the trajectory were shown to be statistically different between fallers and non fallers populations. While these works highlight the effect of different medical conditions on balance, they do not easily 
translate into reliable postural control quantification method, particularly regarding the risk of falling.

More recently, Machine Learning algorithms (and in particular, scoring algorithms) have been applied to attempt to quantify static balance (see e.g. [16], [17], [18]). They show that multivariate approaches (i.e. models using multiple features at the same time, generally through non linear combination) produce significantly better results, and classify fallers and non fallers populations with state of the art performances, with AUC ranging from 0.75 to 0.8 . However, these methods relies on algorithms whose learned models are difficult to interpret from a medical point of view, such as Ranking Forest [19] or local aggregation of Gaussian Mixtures. This is the main drawback of these methods, as in medical applications it is important to understand precisely the results in order to A) interpret them to derive new insights regarding the mechanisms of postural control and B) avoid misinterpretation or "mislearning", as errors can have significant consequences [20].

Contributions. In this work we introduce a new approach to evaluate postural control with learning algorithms, that achieves strong performances while producing models that are easy to understand and interpret. This approach, detailed in Section 3, relies on

- A novel model for postural control and statokinesigrams, from which we derive multiple features,

- A bagging-based algorithm that combines these features while retaining complete interpretability,

- A model-space regularization, based on postural control heuristics, that constrains the search of the optimal classifiers in a restricted manifold and significantly improves the performance of the learning process.

The dataset we used is described in Section 2, and our approach is experimentally studied extensively in Section 4 , where we show that this model outperforms its classical counterparts.

\section{Dataset Acquisition}

We start by introducing the protocol and measurement device that were used for this study.

Measurements. Data acquisition was performed using a Wii Balance Board (Nintendo, Kyoto, Japan) - a low cost force platform that has gained increasing popularity in Posturography in the recent years [21]. For each acquisition, we followed the Romberg's test protocol described in [16]: the participant was asked to stand still on the platform 25 seconds eyes opened then 25 seconds eyes closed, resulting in two statokinesigrams : one open-eyes and one closedeyes. The resulting two dimensional signals (containing the medio-lateral and the antero-posterior coordinates of the CoP over time) were then resampled at $25 \mathrm{~Hz}$, using the SWARII algorithm [22], resulting in signals of length 625 (one example is shown in Figure 1).
Studied populations. In order to test the relevance of our models, we collected data from three different populations, which were ranked according to prior belief about the qualitative nature of their static balance.

- Group 1 : 67 signals recorded from young healthy individuals aged between 20 and 30. This group was assumed to have the best balance, as postural control tends to worsen with age [7].

- Group 2 : 60 signals recorded from healthy elderly individuals, aged between 74 and 86 , which have no medical impairments in balance control and did not report any fall.

- Group 3 : 56 signals recorded from elderly individuals, aged between 74 and 86 , which have declared falls in the past 6 months. This group was assumed to have the worst balance due to the report of falls [23].

It is worth noting that the groups are ordered according to their supposed quality of balance, and that in particular our choice of elderly individual in groups 2 and 3 was in accordance with previous works such as [16], [18], thus allowing to compare our performances to previous works. This data acquisition was part of the clinical study registered at ANSM (ID RCB 2014-A00222-45).

\section{Model and Algorithm}

As stated in the introduction, in order to evaluate postural control from statokinesigrams we use a novel approach that combines A) a new family of descriptors, that can be easily interpreted while providing an efficient ten dimensional embedding of the statokinesigrams, B) a family of white box learning algorithms based on weak one dimensional classifiers and C) a model-space regularization derived from medical heuristics that greatly improved the performances of the weak classifiers. In this section, we start by briefly introducing the model we chose to derive features from statokinesigrams (Section 3.1), and in the second part we present the families of learning algorithm and we discuss the choice and advantages of a model-based regularization approach (Section 3.2).

\subsection{Feature Derivation}

3.1.1. Posturographic Target. Most of the features we derived from statokinesigrams are inspired by the concept of posturographic target (PT) [14]. According to this hypothesis, the movement of the CoP during static balance consists of alternating phases of 1) oscillations around a PT and 2) rapid movement to the next $\mathrm{PT}$.

As the nature of the phases and the positions of the PT are unknown, this model is difficult to use in practice. In this work, we consider a different approach with an unique hypothetical posturographic target (HPT) that continuously moves over time - a first approximation to the original model succession of discrete PTs. To compute the position of the HPT, we assume that 


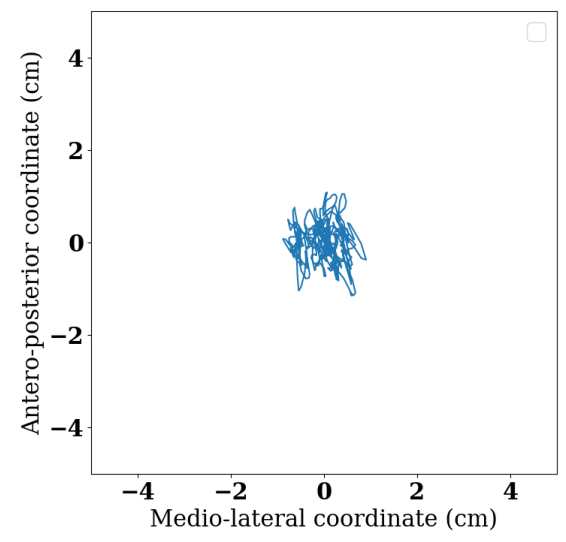

(a) Open-eyes statokinesigram

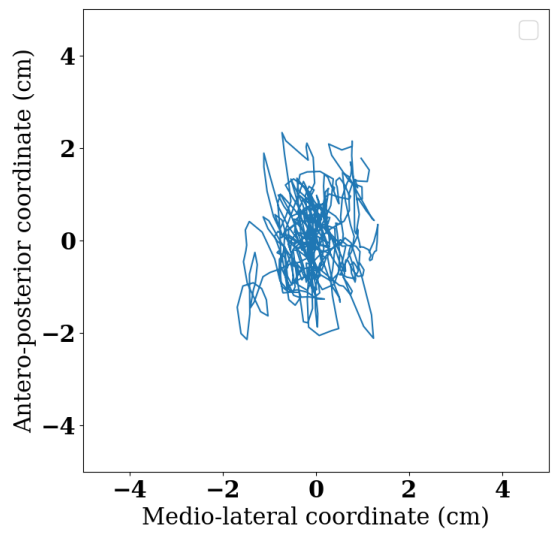

(b) Closed-eyes statokinesigram

Figure 1. Examples of statokinesigrams acquired with the Wii Balance Board, after resampling.

1) the CoP and the HPT start at the same position,

2) the displacements of the HPT are the weighted average of the CoP displacements over a three second window.

In our experiments, the average was computed using geometric weights, with more weight being given to the furthest time in the past. As a result, the HPT displacement is not too sensitive to oscillations or momentary variations of the CoP. Although this model is simple, it yielded good performances in our experiments and naturally led to the definition of the following interpretable features.

3.1.2. HPT-derived Features. From the aforementioned model we derived three groups of features:

- Instability of HPT. These first three features quantify the instability of the HPT, by combining different metrics : the speed of the CoP, the speed of the HPT and the distance between HPT and CoP.

- Excursions. This second group of feature is based on the notion of excursion. We define an excursion as an extended period of time during which the CoP first moves away from the HPT, and then returns close to it. From this concept, we derive the following features

- Remoteness duration: cumulative time during which CoP is moving away from the HPT.

- Return mean time: mean time from which the CoP starts moving closer to the HPT.

- Return mean distance: mean distance from which the CoP starts moving closer to the HPT.

- Dispersion Metrics. This third group includes features that are commonly used in Posturography (see e.g. [24]), namely
- Ellipse Confidence Area: area of the 95\% confidence ellipse containing the entire $\mathrm{CoP}$ trajectory

- Horizontal dispersion: variance of the horizontal coordinate of the CoP.

In total, we derived 16 features ( 8 for both the open-eyes and closed-eyes statokinesigrams) from this model, which are used as the signal embedding for the rest of this work.

\subsection{Scoring Algorithm and Model-space regular- ization}

A key objective of our work was to maximize the interpretability of the learned model while providing accurate evaluation of postural control. This requirement prohibited the use of powerful black box algorithms such as deep learning networks and favored explainable methods such as linear classifiers. Following this idea, we considered the family of learning algorithms based around the combination of easily interpretable weak classifiers (in our case, one dimensional linear support vector machines). These methods include Decision Trees, Adaptive Boosting [25] and Bagging [26].

Another important aspect of our approach is the choice of regularization, as its appropriate use is of paramount importance for learning algorithms, in particular when applied to medical data. Indeed, such datasets tend to be small in size, and usually contain multiple significant sources of noise, such as protocol related inconsistencies, intra-subject variability (tiredness, ..), sensor noise, etc. As a result, even simple models, that are considered more resilient to overfitting, tend to perform poorly if not properly regularized [27].

Commonly used regularizations include Tykhonov / Lasso regularization, or more generally norm-derived penalties $\left(L^{p}\right.$ norms, Schatten norms, ...), as well as different 
approaches such as decision tree pruning. While these regularizations are theoretically sound and powerful off-theshelf tools, in this work we chose to use heuristic based regularizations, that produced significantly better results in our experiments (see Section 4). This process is explained below.

Weak linear classifiers. Let $k \in\{1, \ldots, 16\}$ be the index of one of the features defined in section 3.1. Let $\nu>0$ be the tolerance (a hyperparameter). Let $\mathbf{I}=$ $\left\{x_{i_{1}}^{k}, x_{i_{2}}^{k}, \ldots, x_{i_{n_{1}}}^{k}\right\}$ (resp. $\left.\mathbf{J}=\left\{x_{j_{1}}^{k}, x_{j_{2}}^{k}, \ldots, x_{j_{n_{2}}}^{k}\right\}\right)$ be the values for this feature for the high-quality static balance group (resp. low-quality). Each weak classifier consists in a one dimensional linear SVM, whose hyperplane $\tau \in \mathbb{R}$ (also called threshold) is chosen as the solution of the following optimization problem:

$$
\tau=\arg \max _{t \in \mathbb{R}} v(t)
$$

where $v(t)$ is the value of a threshold and is defined as

$$
v(t)=\max \left\{v_{\text {left }}(t), v_{\text {right }}(t)\right\}
$$

with

$v_{\text {left }}(t)=\left\{\begin{array}{l}0 \text { if } \sum_{x_{\ell}^{k} \in J} \mathbb{1}\left\{x_{\ell}^{k}<t\right\}>\frac{n_{1}}{n_{2}} \nu \\ \frac{n_{2}}{n_{1}} \sum_{x_{\ell}^{k} \in I} \mathbb{1}\left\{x_{\ell}^{k}<t\right\}-\sum_{x_{\ell}^{k} \in J} \mathbb{1}\left\{x_{\ell}^{k}<t\right\} \text { otherwise }\end{array}\right.$

$v_{\text {right }}(t)=\left\{\begin{array}{l}0 \text { if } \sum_{x_{\ell}^{k} \in I} \mathbb{1}\left\{x_{\ell}^{k}>t\right\}>\frac{n_{2}}{n_{1}} \nu \\ \sum_{x_{\ell}^{k} \in J} \mathbb{1}\left\{x_{\ell}^{k}>t\right\}-\frac{n_{2}}{n_{1}} \sum_{x_{\ell}^{k} \in I} \mathbb{1}\left\{x_{\ell}^{k}>t\right\} \text { otherwise }\end{array}\right.$

represent respectively the left and right values of the threshold. Note that the tolerance $\nu$ restricts the number of missclassified individuals below (resp. above) the threshold in $v_{\text {left }}$ (resp. $\left.v_{\text {right }}\right)$. Since the value $v$ is piece-wise constant, the maximum is not unique, and in this case we chose $\tau$ following the principle of margin maximization.

The value function $v$ varies from the traditional objective function of linear classifiers as it enforces that the values of the feature from the high-quality postural control population should be smaller than the threshold while low-quality static balance related values should be greater - breaking the symmetry of the threshold. By limiting the number of possible classifiers, this constraint acts as a model-space regularization. This particular limitation also reflects the heuristic that, for the features defined in Section 3.1, individuals with better static balance generally present lower values than their worse counterpart. These one dimensional classifiers are used in the three aforementioned learning algorithms: as weak classifiers for both Adaptive Boosting and Bagging, and as node decision rules of Decision Trees.
Heuristic Based Bagging. We also considered a variation of the classical bagging algorithm by using additional heuristic derived constraints. As the open-eyes and the closed-eyes parts of the protocol are different exercices involving different mechanisms of postural control [15], we assumed that they contained complementary information regarding static balance and we constrained the bagging algorithm selection of feature to choose five open-eyes features and five closed-eyes features. A weak classifier is then trained on each of the selected features.

Heuristic Based Decision Trees. In addition to regular decision tree and random forest algorithms, we also used a decision forest algorithm that embedded known posturography heuristics. Following the same idea as for the Heuristic Based Bagging, the forest was constrained to contain two trees, one trained on open-eyes features and one of closed-eyes features. Additionally, we replaced the tree pruning by the following rule: after each split, one of the child node was constrained to be terminal: the left child if the threshold was selected using its $v_{\text {left }}$, or the right child otherwise.

\section{Experimental Evaluations}

In this section we present the experiments we conducted to test algorithms introduced in section 3 .

\subsection{Learning Algorithms Comparison}

This experiment aimed to observe how the different learning algorithms considered in its paper perform, particularly compared to their more usual counterpart. We evaluated the five following algorithms :

- 1. RF - Random Forest

- 2. Adaboost - Adaboost with Decision Trees

- 3. HBagging - Heuristic based bagging (Section 3)

- 4. HDTrees - Heuristic based Decision Trees (Section 3)

- 5. HAdaboost - Adaboost with the heuristic based Weak Classifier (Section 3)

In all the experiments we set $\nu=0.1$, while RF and Adaboost hyperparameters were chosen by cross validation. Each algorithm was evaluated on each pair of groups. When compared together, group 1 and 2 were labeled 0 and 1 respectively, group 1 and 3 were labeled 0 and 1 and group 2 and 3 were labeled 0 and 1 (in this case 0 stands for better postural control and 1 for worse). For each pair of groups, the data was split into train and test (70\%-30\%). The AUC of the algorithms on the test case was averaged over 50 random train test split.

Results are reported in Table 1. All the algorithms show better performances when the groups to separate are more distant in term of balance (group 1 and group 3). This is coherent with our thought that the models capture the degradation of balance. Overall the algorithms using the heuristic based regularization appear to outperform their classical 


\begin{tabular}{l|ccc} 
Groups & $1-2$ & $2-3$ & $1-3$ \\
\hline RF & $0.70( \pm 0.08)$ & $0.65( \pm 0.06)$ & $0.85( \pm 0.04)$ \\
\hline Adaboost & $0.74( \pm 0.06)$ & $0.60( \pm 0.08)$ & $0.86( \pm 0.06)$ \\
\hline HBagging & $0.72( \pm 0.07)$ & $0.75( \pm 0.07)$ & $0.90( \pm 0.05)$ \\
\hline HDTrees & $0.69( \pm 0.08)$ & $0.70( \pm 0.07)$ & $0.88( \pm 0.05)$ \\
\hline HAdaboost & $0.69( \pm 0.07)$ & $0.73( \pm 0.08)$ & $0.90( \pm 0.05)$ \\
\hline
\end{tabular}

TABLE 1. MEAN AND STANDARD DEVIATION OF AUC OF THE DIFFERENT ALGORITHMS FOR EACH PAIR OF GROUPS.

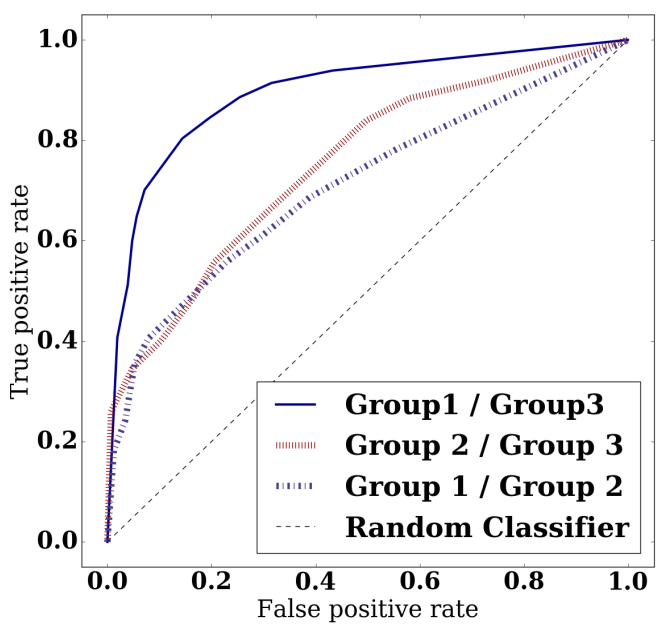

Figure 2. ROC curves for HBagging

counterparts, despite RF and Adaboost hyperparameters being tuned with cross validation. This result highlights the importance of proper regularization strategies, and might also suggest that carefully designed constraints, if accessible, are better than off-the-shelf regularization. Finally, it is interesting to note that the algorithm with the best global performance was HBagging, which is also arguably the easiest to interprete as it just compute the average values of the weak classifiers. Figure 2 shows the ROC curves for each pair of group for HBagging.

\subsection{Comparing Population Scores}

This experiment aimed to verify if the quantification of balance computed by HBagging was coherent with the qualitative evaluation of the different groups. To test this hypothesis, we proceeded as follows: group 1 (i.e. individuals with the best postural control) was labeled as class 0 while group 3 (the worst group) was labeled as class 1 . Elements of group 1 and group 3 were randomly split into train and test (50\%-50\%). After training, the model was used to compute the score of each individual in the test set of group 1 and group 3, and in the entire set of group 2. We then compared the mean and standard deviation of

\begin{tabular}{l|ccc} 
Group & 1 & 2 & 3 \\
\hline Mean(std) & $0.09( \pm 0.14)$ & $0.23( \pm 0.21)$ & $0.37( \pm 0.21)$ \\
\hline TABLE 2. MEAN AND STANDARD DEVIATION OF GROUP SCORES.
\end{tabular}

each group. This experiment was repeated using 10 different random train test splits.

Results are reported in Table 2. It can be seen that the scores increase with the expected qualitative degradation of balance.

\section{Discussion}

Heuristic based models. As illustrated by the results of Table 1, heuristic based learning algorithms and regularizations outperform their regular counterparts while maximizing the resulting model interpretability. This remark encourages further works in this direction.

Score Interpretation. An advantage of Hbagging is the interpretability of the score. In each dimension, the weak classifier provides a class prediction. If the prediction is one, it means that the subject has a statokinesigram classified as abnormal is this dimension. The final score is the average of the scores obtained with the open-eyes classifier and the closed-eyes classifier. Therefore the final score represents the proportion of dimensions with values larger than the one of control population. Higher scores suggest impairments in postural control and therefore increased risk of falling.

Limitation of the model. Our model may not fully and accurately quantify postural control during the Romberg's test. Interpretability is therefore crucial to detect weaknesses in the model in order to A) provide context to the results and B) permit meaningful discussions with medical specialists for continuous adjustements of the features and heuristics. For example in our model the statokinesigram of an individual affected by Parkinson's disease may be given a low score, although it should be classified as abnormal as the individual has an impaired balance. It means that our features and heuristics do not capture yet all the nuances in balance degradation.

Shortcoming in our model include also the lack of local information about the movement of the $\mathrm{CoP}$ as all features consist of trajectory-long averages. Therefore, an interesting future research direction would be to adapt our model along the lines developped in [18].

It is crucial to note that our model's score alone does not give enough information for a proper diagnosis. In particular it does not take into account eventual particularities of the protocol sequence, for example slight deviation from the theoretical protocol or stress during experiment, or other knowledge about the individual that may change completely the interpretation of the score.

\section{Acknowledgments}

The authors would like to thank Damien Ricard (COGNAG-G UMR 8257, CNRS, Ecole du Val-de-Grâce, 
Service de Neurologie, HIA Percy, SSA, France) for its invaluable help in collecting the statokinesigrams. This work was partly supported by the Swiss National Science Foundation under grant number 407540_167320.

\section{References}

[1] J. Kober, J. A. Bagnell, and J. Peters, "Reinforcement learning in robotics: A survey," The International Journal of Robotics Research, vol. 32, no. 11, pp. 1238-1274, 2013.

[2] I. Goodfellow, Y. Bengio, A. Courville, and Y. Bengio, Deep learning. MIT press Cambridge, 2016, vol. 1.

[3] K. Christensen, G. Doblhammer, R. Rau, and J. W. Vaupel, "Ageing populations: the challenges ahead," The lancet, vol. 374, no. 9696, pp. 1196-1208, 2009.

[4] K. Sirinukunwattana, S. E. A. Raza, Y.-W. Tsang, D. R. Snead, I. A. Cree, and N. M. Rajpoot, "Locality sensitive deep learning for detection and classification of nuclei in routine colon cancer histology images," IEEE transactions on medical imaging, vol. 35, no. 5, pp. 1196-1206, 2016.

[5] M. Li and Z.-H. Zhou, "Improve computer-aided diagnosis with machine learning techniques using undiagnosed samples," IEEE Transactions on Systems, Man, and Cybernetics-Part A: Systems and Humans, vol. 37, no. 6, pp. 1088-1098, 2007.

[6] R. W. Sattin, D. A. LAMBERT HUBER, C. A. DEVITO, J. G. RODRIGUEZ, A. ROS, S. BACCHELLI, J. A. Stevens, and R. J. Waxweiler, "The incidence of fall injury events among the elderly in a defined population," American journal of epidemiology, vol. 131, no. 6, pp. 1028-1037, 1990.

[7] M. E. Tinetti, "Preventing falls in elderly persons," New England journal of medicine, vol. 348, no. 1, pp. 42-49, 2003.

[8] M. Van Diest, C. J. Lamoth, J. Stegenga, G. J. Verkerke, and K. Postema, "Exergaming for balance training of elderly: state of the art and future developments," Journal of neuroengineering and rehabilitation, vol. 10, no. 1, p. 101, 2013.

[9] R. J. Peterka and M. S. Benolken, "Role of somatosensory and vestibular cues in attenuating visually induced human postural sway," Experimental brain research, vol. 105, no. 1, pp. 101-110, 1995.

[10] P. P. Perrin, C. Jeandel, C. A. Perrin, and M. C. Bene, "Influence of visual control, conduction, and central integration on static and dynamic balance in healthy older adults," Gerontology, vol. 43, no. 4, pp. 223-231, 1997.

[11] I. Melzer, N. Benjuya, and J. Kaplanski, "Postural stability in the elderly: a comparison between fallers and non-fallers," Age and ageing, vol. 33, no. 6, pp. 602-607, 2004.

[12] B. J. Benda, P. O. Riley, and D. E. Krebs, "Biomechanical relationship between center of gravity and center of pressure during standing," IEEE Transactions on Rehabilitation Engineering, vol. 2, no. 1, pp. 3-10, 1994.

[13] M. Piirtola and P. Era, "Force platform measurements as predictors of falls among older people-a review," Gerontology, vol. 52, no. 1, pp. 1-16, 2006.

[14] L. Baratto, P. G. Morasso, C. Re, and G. Spada, "A new look at posturographic analysis in the clinical context: sway-density versus other parameterization techniques," Motor control, vol. 6, no. 3, pp. 246-270, 2002.

[15] A. Khasnis, R. Gokula et al., "Romberg's test." Journal of postgraduate medicine, vol. 49, no. 2, p. 169, 2003.

[16] J. Audiffren, I. Bargiotas, N. Vayatis, P.-P. Vidal, and D. Ricard, "A non linear scoring approach for evaluating balance: classification of elderly as fallers and non-fallers," PLoS one, vol. 11, no. 12, p. e0167456, 2016.
[17] C.-L. Liu, C.-H. Lee, and P.-M. Lin, "A fall detection system using knearest neighbor classifier," Expert systems with applications, vol. 37, no. 10 , pp. 7174-7181, 2010.

[18] I. Bargiotas, J. Audiffren, N. Vayatis, P.-P. Vidal, S. Buffat, A. P. Yelnik, and D. Ricard, "On the importance of local dynamics in statokinesigram: A multivariate approach for postural control evaluation in elderly," PloS one, vol. 13, no. 2, p. e0192868, 2018.

[19] S. Clémençon, M. Depecker, and N. Vayatis, "Ranking forests," Journal of Machine Learning Research, vol. 14, no. Jan, pp. 39-73, 2013.

[20] T. Ching, D. S. Himmelstein, B. K. Beaulieu-Jones, A. A. Kalinin, B. T. Do, G. P. Way, E. Ferrero, P.-M. Agapow, M. Zietz, M. M. Hoffman et al., "Opportunities and obstacles for deep learning in biology and medicine," bioRxiv, p. 142760, 2018.

[21] R. A. Clark, A. L. Bryant, Y. Pua, P. McCrory, K. Bennell, and M. Hunt, "Validity and reliability of the nintendo wii balance board for assessment of standing balance," Gait \& posture, vol. 31, no. 3, pp. 307-310, 2010.

[22] J. Audiffren and E. Contal, "Preprocessing the nintendo wii board signal to derive more accurate descriptors of statokinesigrams," Sensors, vol. 16 , no. 8, p. 1208, 2016.

[23] G. F. Fuller, "Falls in the elderly." American family physician, vol. 61, no. 7, pp. 2159-68, 2000.

[24] R. J. Doyle, E. T. Hsiao-Wecksler, B. G. Ragan, and K. S. Rosengren, "Generalizability of center of pressure measures of quiet standing," Gait \& posture, vol. 25, no. 2, pp. 166-171, 2007.

[25] Y. Freund and R. E. Schapire, "A decision-theoretic generalization of on-line learning and an application to boosting," Journal of computer and system sciences, vol. 55, no. 1, pp. 119-139, 1997.

[26] L. Breiman, "Bagging predictors," Machine learning, vol. 24, no. 2, pp. 123-140, 1996.

[27] B. Schölkopf and A. J. Smola, Learning with kernels: support vector machines, regularization, optimization, and beyond. MIT press, 2002 